\title{
CHARACTERIZATION OF THE FINITE PARTITION PROPERTY FOR A COLLECTION OF UNIVERSAL SUBCONTINUA
}

\author{
WILLIAM T. TROTTER, JR.
}

Let $X$ be a Hausdorff space and $A \subset X$ a continuum. $A$ is said to be a Universal Subcontinuum (USC) if $A \cap B$ is connected for every continuum $B \subset X$. Let $\alpha$ be a collection of USC's of a Hausdorff space. Then $\alpha$ is said to have the finite partition property if $\alpha$ has a decomposition into a finite number of subcollections each having the finite intersection property. A result due to $\mathrm{W}$. J. Gray [1] can be easily modified to show that in a Hausdorff space, a collection of USC's has the finite intersection property if every pair has a common point. Other properties of USC's are given in [2] and [3].

Theorem. Let $\alpha$ be a collection of USC's of a Hausdorff space. Then the following statements are equivalent.

(1) $\alpha$ has the finite partition property.

(2) There exist integers $p, q$ with $p \geqq q \geqq 2$ such that for every $p$ elements of $\alpha$, at least $q$ of them have a common point.

(3) $\alpha$ has no infinite pairwise disjoint subcollection.

Proof. (1) implies (2) and (2) implies (3) are obvious. Condition (2) is used in [2] to obtain a result which states that the maximal number of subcollections required for the partition is $p-q+2$. We now prove that (3) implies (1). The proof is by contradiction; we assume that $\alpha$ is a collection of USC's of a Hausdorff space with no infinite pairwise disjoint subcollection, but that $\alpha$ does not have the finite partition property.

Let $I(\alpha)=\{\beta \subset \alpha \mid \beta$ is pairwise disjoint $\}$. Let $\beta_{1}, \beta_{2} \in I(\alpha)$. We say $\beta_{1} \leqq \beta_{2}$ if $\beta_{1} \subset \beta_{2}$. Then it is clear that $\leqq$ defines a partial order on $I(\alpha)$. Let $\left\{\beta_{j} \mid j \in J\right\}$ be a totally ordered subset of $I(\alpha)$. Then define $\beta=\bigcup_{j \in J} \beta_{j}$. We show $\beta \in I(\alpha)$. Let $H, G \in \beta$, then there exist $j_{1}$, $j_{2} \in J$ such that $H \in \beta_{j_{1}}$ and $G \in \beta_{j_{2}}$. We may assume $\beta_{j_{1}} \subset \beta_{j_{2}}$ which implies that $H, G \in \beta_{j_{2}}$ and that $H \cap G=\varnothing$ if $H \neq G$. Thus $\beta$ is pairwise disjoint and $\beta \in I(\alpha)$. It is clear that $\beta \geqq \beta_{j}$ for every $j \in J$ and hence $\beta$ is an upper bound for the chain. Thus every pairwise disjoint subcollection of $\alpha$ is a subset of some maximal element of $I(\alpha)$.

Let $\alpha=\alpha_{0}$; we define the following subcollections inductively: $\beta_{i}, \alpha_{i}^{1}, \alpha_{i}^{2}, \beta_{i}^{1}, \alpha_{i}^{3}, \alpha_{i+1}$.

Received by the editors May 9, 1969. 
(1) $\beta_{i}$ is a maximal element of $I\left(\alpha_{i}\right)$ with card $\beta_{i} \geqq 2$.

(2) $\alpha_{i}^{1}=\left\{H \in \alpha_{i} \mid\right.$ there exist $B_{1}, B_{2} \in \beta_{i}$ such that $B_{1} \cap B_{2}=\varnothing$ and $\left.H \cap B_{1} \neq \varnothing \neq H \cap B_{2}\right\}$.

(3) $\alpha_{i}^{2}=\left\{H \in \alpha_{i}-\alpha_{i}^{1} \mid\right.$ there exists $B_{1}, B_{2} \in \beta_{i}$ and $G \in \alpha_{i}-\alpha_{i}^{1}$ such that $B_{1} \cap B_{2}=\varnothing, H \cap B_{1} \neq \varnothing \neq G \cap B_{2}$, and $\left.H \cap G \neq \varnothing\right\}$.

(4) $\beta_{i}^{1}=\left\{B \in \beta_{i} \mid\right.$ there exist $G_{B}, H_{B} \in \alpha_{i}-\alpha_{i}^{1}-\alpha_{i}^{2}$ such that $G_{B} \cap B$ $\neq \varnothing \neq H_{B} \cap B$ and $\left.G_{B} \cap H_{B}=\varnothing\right\}$.

(5) $\alpha_{i}^{3}=\left\{H \in \alpha_{i}-\alpha_{i}^{1}-\alpha_{i}^{2} \mid\right.$ there exists $B \in \beta_{i}-\beta_{i}^{1}$ such that $H \cap B$ $\neq \varnothing\}$.

(6) $\alpha_{i+1}=\alpha_{i}-\alpha_{i}^{1}-\alpha_{i}^{2}-\alpha_{i}^{3}$.

We proceed as follows: Since $\alpha_{0}$ does not have the finite intersection property, there exist $H, G \in \alpha_{0}$ such that $H \cap G=\varnothing$. Then there exists a maximal element $\beta_{0} \in I\left(\alpha_{0}\right)$ with $\{H, G\} \leqq \beta_{0}$. We now show that $\alpha_{0}^{1}$ and $\alpha_{0}^{2}$ have the finite partition property.

We associate each element of $\alpha_{0}^{1}$ with the disjoint pair $B_{1}, B_{2} \in \beta_{0}$ given in the definition of $\alpha_{0}^{1}$ and then show that the collection of all elements associated with the same pair has the finite intersection property. Let $H_{1}, H_{2} \in \alpha_{0} ; B_{1}, B_{2} \in \beta_{0} ; H_{1} \cap B_{1} \neq \varnothing \neq H_{1} \cap B_{2} ; H_{2} \cap B_{1}$ $\neq \varnothing \neq H_{2} \cap B_{2}$; and $B_{1} \cap B_{2}=\varnothing$. Suppose $H_{1} \cap H_{2}=\varnothing$. Then $B_{1} \cup H_{1}$ $\cup B_{2}$ and $B_{1} \cup H_{2} \cup B_{2}$ are USC's but $\left(B_{1} \cup H_{1} \cup B_{2}\right) \cap\left(B_{1} \cup H_{2} \cup B_{2}\right)$ $=B_{1} \cup B_{2}=B_{1} \mid B_{2}$. The contradiction shows $H_{1} \cap H_{2} \neq \varnothing$. By hypothesis, card $\beta_{0}$ is finite and it is clear that $\alpha_{0}^{1}$ has the finite partition property. The proof that $\alpha_{0}^{2}$ has the finite partition property is similar.

Now suppose that $\beta_{0}^{1}=\varnothing$. Then since $\beta_{0}$ is maximal, every $H \in \alpha_{0}$ intersects some $B \in \beta_{0}$. We then decompose $\alpha_{0}-\alpha_{0}^{1}-\alpha_{0}^{2}$ into at most card $\beta_{0}$ subcollections by associating each element with the unique $B \in \beta_{0}$ which it intersects. Since $\beta_{0}^{1}=\varnothing$, every pair associated with the same $B$ have a common point. Thus $\alpha_{0}-\alpha_{0}^{1}-\alpha_{0}^{2}$ has the finite partition property and hence so does $\alpha_{0}$. The contradiction shows $\beta_{0}^{1} \neq \varnothing$. It is clear that $\alpha_{0}^{3}$ has the finite partition property and that $\left\{G_{B}, H_{B} \mid B \in \beta_{0}^{1}\right\} \in I\left(\alpha_{1}\right)$. We choose a maximal element $\beta_{1} \in I\left(\alpha_{1}\right)$ such that $\left\{G_{B}, H_{B} \mid B \in \beta_{0}^{1}\right\} \leqq \beta_{1}$. It $\alpha_{1}$ has the finite partition property, then so does $\alpha_{0}=\alpha_{1} \cup \alpha_{0}^{1} \cup \alpha_{0}^{2} \cup \alpha_{0}^{3}$. Therefore we assume that $\alpha_{1}$ does not have the finite partition property, and it is clear that this argument can be repeated inductively.

We now show that the sequence $\left\{\beta_{i}\right\}$ satisfies the following property:

(p) Suppose $i, j$, and $k$ are integers such that $i \geqq j$ and $i \geqq k$. Let $B_{i} \in \beta_{i}, \quad B_{j} \in \beta_{j}$, and $B_{k} \in \beta_{k}$. Then $B_{i} \cap B_{j} \neq \varnothing \neq B_{i} \cap B_{k}$ implies $B_{j} \cap B_{k} \neq \varnothing$.

Assume $B_{j} \cap B_{k}=\varnothing$. If $i=j$, then $B_{i}=B_{j}$ and $B_{j} \cap B_{k} \neq \varnothing$. Thus 
$i \neq j$ and similarly, we have $i \neq k$. If $j=k$, then $B_{j} \cap B_{k}=\varnothing$ implies $B_{i} \in \alpha_{j}^{1}$. Thus $j \neq k$ and we may assume $i>j>k$. Since $B_{j} \in \alpha_{j} \subset \alpha_{k}$ and $\beta_{k}$ is a maximal element of $I\left(\alpha_{k}\right)$, there exists $H_{k} \in \beta_{k}$ such that $B_{j} \cap H_{k} \neq \varnothing$ and $H_{k} \cap B_{k}=\varnothing$. But this implies $B_{j} \in \alpha_{k}^{2}$. The contradiction shows $B_{j} \cap B_{k} \neq \varnothing$.

Let $\gamma_{i}=U_{B \in \beta_{i}} B$. We now show $\bigcap_{i=0}^{\infty} \gamma_{i} \neq \varnothing$. Since each $\gamma_{i}$ is compact, it suffices to show $\bigcap_{i=0}^{k} \gamma_{i} \neq \varnothing$ in every $k \geqq 0$. Let $B_{k} \in \beta_{k}$. Then in every $i \leqq k$, we have $B_{k} \in \alpha_{k} \subset \alpha_{i}$ and since $\beta_{i}$ is a maximal element of $I\left(\alpha_{i}\right)$, there exists $B_{i} \in \beta_{i}$ such that $B_{k} \cap B_{i} \neq \varnothing$. But property (p) obviously implies $\bigcap_{i=0}^{k} B_{i} \neq \varnothing$ and thus $\bigcap_{i=0}^{\infty} \gamma_{i} \neq \varnothing$.

Let $x \in \cap_{i=0}^{\infty} \gamma_{i}$. Then for every $i \geqq 0$, there exists $D_{i} \in \beta_{i}$ such that $x \in D_{i}$. We now define a collection of USC's $\left\{E_{i} \mid i \geqq 0\right\}$ as follows:

(i) Since card $\beta_{0} \geqq 2$, there exists $E_{0} \in \beta_{0}$ such that $E_{0} \cap D_{0}=\varnothing$.

(ii) For every $i \geqq 0, \quad D_{i+1} \in \alpha_{i}-\alpha_{i}^{1}-\alpha_{i}^{2}-\alpha_{i}^{3}$ and $D_{i+1} \cap D_{i} \neq \varnothing$ imply $D_{i} \in \beta_{i}^{1}$. Therefore, there exists $E_{i+1} \in \beta_{i+1}$ such that $E_{i+1}$ $\cap D_{i} \neq \varnothing$ but $E_{i+1} \cap D_{i+1}=\varnothing$.

We now show that the infinite collection $\left\{E_{i} \mid i \geqq 0\right\}$ is pairwise disjoint. The proof is by induction. We have $E_{0} \cap D_{0}=\varnothing, E_{1} \cap D_{0}$ $\neq \varnothing, D_{1} \cap D_{0} \neq \varnothing$, and $E_{1} \cap D_{1}=\varnothing$. Therefore $\left\{E_{0}, E_{1}, D_{1}\right\}$ is pairwise disjoint. Now assume $\left\{E_{0}, E_{1}, \cdots, E_{k}, D_{k}\right\}$ is pairwise disjoint. Then since $D_{k+1} \cap D_{k} \neq \varnothing, E_{k+1} \cap D_{k} \neq \varnothing$, and $E_{k+1} \cap D_{k+1}=\varnothing$, property (p) implies $\left\{E_{0}, E_{1}, \cdots, E_{k}, E_{k+1}, D_{k+1}\right\}$ is pairwise disjoint. By induction, $\left\{E_{i} \mid i \geqq 0\right\}$ is an infinite pairwise disjoint subcollection of $\alpha$. This contradiction completes the proof.

\section{REFERENCES}

1. W. J. Gray, A fixed point theorem for commuting monotone functions, Canad. J. Math 21 (1969), 502-504.

2. W. T. Trotter, A decomposition theorem for collections of universal subcontinua, Colloq. Math. (to appear).

3. A. D. Wallace, Monotone transformations, Duke Math J. 9 (1942), 487-506, MR $4,147$.

University of Alabama, University, Alabama 35486 